\title{
Reviews in neuroimmunology
}

\author{
Robert S. Fujinami
}

Received: 18 December 2013 / Accepted: 23 December 2013 / Published online: 19 February 2014

(C) Journal of NeuroVirology, Inc. 2014

This special issue highlights young investigators in the area of neurovirology. They represent a new wave of researchers who will carry the field into the future. We are delighted to feature some of their works in this special issue. The work spans a wide range of virus-CNS-host interactions, and they represent the field well.

Aaron Johnson is at the Mayo Clinic and provides us with insights into antiviral CD8 $+\mathrm{T}$ cells and the disruption of the blood brain barrier (BBB). He spent most of his early training at the Mayo Graduate School and received his $\mathrm{PhD}$ there in Biomedical Science. His postdoctoral training was at the Mayo Clinic and at the University of Washington.

Brenda Fredericksen is at the University of Maryland. She studies models of flavivirus pathogenesis. She received her $\mathrm{PhD}$ at the University of Tennessee Health Science Center and received additional training at Washington University. She provides us with an excellent review of West Nile Virus pathogenesis.

Jayasri Das Sarma received her early training in India and much of her scientific career in Philadelphia. She is currently at the Thomas Jefferson University in Philadelphia. Jayasri provides an interesting look at microglial cells and interactions with mouse hepatitis virus. Her work shows that microglial cells can amplify the virus-induced disease.

Jennifer Gordon spent her education and training in Pennsylvania. She received her PhD from Drexel (MCP Hahnemann) School of Medicine and postdoctoral fellowship at Temple. She is at Temple University. One of her areas of expertise is human JC virus (JCV). She provides an excellent review of the contributions of the immune response to JCV and how immunomodulatory therapies can lead to the development of progressive multifocal leukoencephalopathy.

R. S. Fujinami $(\bowtie)$

University of Utah, Salt Lake City, UT, USA

e-mail: Robert.Fujinami@hsc.utah.edu

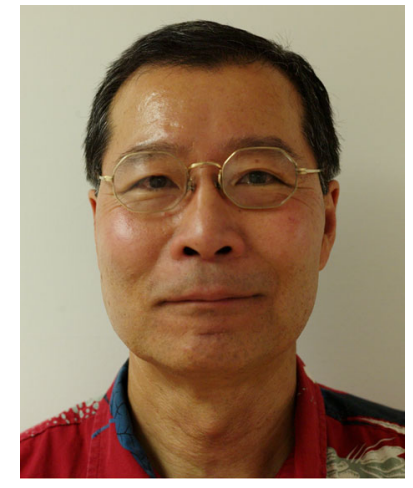

Julie Olson is at the University of Minnesota. She received her $\mathrm{PhD}$ from the University of Iowa College of Medicine. Julie spent additional training at Northwestern University. She provides a review about the innate immune response to viral infection and contribution to demyelinating disease.

Karin Peterson is at the Rocky Mountain Laboratories (RML) in Hamilton, Montana. Karin received her PhD from the University of Missouri Medical School. She then went to RML as a postdoctoral fellow, studying retrovirus pathogenesis in the CNS. She started out as faculty at LSU and later returned to RML. Karin provides a review of La Crosse virus pathogenesis in the CNS and innate contributions to disease.

Maria Nagel received her MS from the University of Illinois and took additional training at the University of Colorado School of Medicine where she is now on the faculty. She provides new findings on varicella zoster virus (VZV) vasculopathy. This is a newly appreciated area of exploration in VSV pathogenesis.

Mineki Saito is at the Kawasaki Medical School where he studies HTLV-1 pathogenesis. He provides a review of adult T cell leukemia/lymphoma (ATL) and HTLV-1 associated myelopathy/tropical spastic paraparesis (HAM/TSP). Various 
aspects of the innate and adaptive immune response to the virus contribute to the pathogenesis.

Pooja Jain is at the Drexel Institute for Biotechnology and Virology Research. She received her PhD from the Central Drug Research Institute in India. She, with Shilpa Buch who is at the Nebraska Medical Center in Omaha, provides new and exciting work on the role of dendritic cells in the pathogenesis of neuroAIDS.
Xuebin Qin received his MD at the Wannan Medical College in Wuhu China and $\mathrm{PhD}$ at the Peking Union Medical College and Chinese Academy of Medical Sciences in Bejing, China. After obtaining additional training in Boston at Tufts University and Harvard Medical School, he is now at Temple University. Part of his research is studying CD59 and HIV lysis. This review summarizes some of this work. 